\title{
Perspective
}

PERSPECTIVE Actualité en histoire de l'art

$2 \mid 2020$

Danser

\section{Pietra viva : mouvement implicite, semblant de vie et anthropologie de l'art}

Pietra viva: Implicit Movement, Semblance of Life and Anthropology of Art

Pietra viva: Implizite Bewegung, täuschende Lebendigkeit und Anthropologie

der Kunst

Pietra viva: movimento implicito, parvenza di vita e antropologia dell'arte

Pietra viva: movimiento implícito, semblante de vida y antropología del arte

\section{Carlo Severi}

\section{OpenEdition}

Journals

\section{Édition électronique}

URL : https://journals.openedition.org/perspective/20676

DOI : $10.4000 /$ perspective. 20676

ISSN : 2269-7721

Éditeur

Institut national d'histoire de l'art

\section{Édition imprimée}

Date de publication : 30 décembre 2020

Pagination : 143-154

ISBN : 978-2-917902-90-5

ISSN : $1777-7852$

Référence électronique

Carlo Severi, «Pietra viva : mouvement implicite, semblant de vie et anthropologie de l'art », Perspective [En ligne], 2 | 2020, mis en ligne le 30 juin 2021, consulté le 31 juillet 2022. URL : http:// journals.openedition.org/perspective/20676; DOI : https://doi.org/10.4000/perspective.20676 


\title{
Pietra viva : mouvement implicite, semblant de vie et anthropologie de l'art
}

\author{
Carlo Severi
}

Negli anni molti e nelle molte pruove, cercando, il saggio al buon concetto arriva d'un'immagine viva, vicino a morte, in pietra alpestra e dura ${ }^{1}$

Comment "danse " une peinture ? Comment peut-elle sembler "vivante " ? Le thème du mouvement implicite attribué à un corps peint est ancien. Dans le livre II du De Pictura, Leon Battista Alberti écrit que la perception du mouvement physique des figures est indispensable à la perception de ce " mouvement de l'âme » vers laquelle toute peinture doit tendre ${ }^{2}$. Ainsi la perspective consisterait-elle non seulement en un déchiffrement de la profondeur à partir d'une surface, mais aussi en la compréhension d'une dynamique implicite, propre à l'action attribuée aux figures. Alberti ajoute qu'un corps ne peut suggérer le mouvement que s'il engendre l'illusion d'un être vivant ${ }^{3}$. La notion de mouvement suppose donc, à ses yeux, l' " apparence de la vie ", ce parer vivo qui joue un rôle crucial dans la conception de la perspective italienne dès le Quattrocento. C'est à partir de deux ouvres de Mantegna, le Saint Sébastien de la Ca' d'Oro, à Venise, et L'Introduction du culte de Cybèle à Rome, dans lesquelles les thèmes du mouvement implicite et de l'attribution d'une apparence de vie aux figures jouent un rôle particulièrement significatif, que je souhaite mener une réflexion sur le mouvement implicite et la notion de vie de l'image dans la perspective italienne du Quattrocento.

Comment un anthropologue peut-il prétendre étudier de tels chefs-d'œuvre de l'art occidental ? Comment définir son point de vue dans ce contexte ? Quels résultats peut-il ou elle espérer, qui ne soient pas déjà connus des historiens de l'art ? J'ai constaté, il y a une trentaine d'années ${ }^{4}$, qu'un étrange paradoxe semblait peser sur l'anthropologie de l'art. Alors que les anthropologues tentent de démontrer la validité de leur discipline pour étudier n'importe quelle société, traditionnelle ou moderne, les spécialistes en anthropologie de l'art se consacrent aujourd'hui encore presque exclusivement aux arts non occidentaux ou aux formes "populaires", " pathologiques " ou infantiles des arts européens. Je me suis demandé pourquoi ces derniers n'approchaient le " grand art " de l'Occident, ses courants d'idées, ses enjeux, ses grands artistes qu'en de très rares occasions, alors que de nombreux historiens de l'art occidental, à partir d'Aby Warburg, évidemment - mais je pense aussi à Michael Baxandall, David Freedberg, Salvatore Settis, Hans Belting, Caroline 
van Eck ou Horst Bredekamp - se sont rapprochés de l'anthropologie pour renouveler leurs approches. J'ai reconnu ailleurs que les causes de ce paradoxe sont multiples ${ }^{5}$, et les limites imposées à cet article ne me permettent pas d'entrer dans les détails de ce débat. Esquissons donc brièvement l'essentiel de notre approche, qui consiste à passer de l'étude du sens des œuvres (ou de leur iconographie) à l'exploration de la relation imaginaire qu'elles entretiennent avec l'observateur. On se propose donc de passer d'un regard analytique et historique à l'étude de ce qu'Aby Warburg, dans ses Fragments sur l'expression, a appelé le " regard anthropomorphe ${ }^{6}$ " que l'on peut poser sur une œuvre d'art. De ce point de vue, le parer vivo (ou " semblant de vie ") apparaît comme une forme spécifique d'agentivité de la perspective. Profondément lié à l'idée de perspective, le parer vivo ne se confond ni avec les règles géométriques de composition de l'image, ni avec le déchiffrement de la profondeur, ni avec la seule interprétation du mouvement implicite des figures. Pour Alberti comme pour Ernst Gombrich ou Thomas Kuhn ${ }^{7}$ de nos jours, la perspective est avant tout une science qui applique les lois de l'optique et de la géométrie à la représentation de l'espace. Elle réalise ainsi ce que l'on pourrait appeler une vérité visuelle de la peinture et participe du même idéal épistémologique que la science moderne. Le parer vivo - le principe de l'apparence du vivant - est au contraire une forme spécifique d'illusion. Plus précisément, il désigne l'effet de réel qu'une telle illusion est susceptible de déclencher, et qui implique non seulement la construction d'un espace vraisemblable mais aussi la perception d'une vie des figures dont, comme on vient de le voir, le mouvement implicite est un élément constitutif. Dans L'objet-personne, j'ai essayé de montrer qu'à partir de l'épistémologie de cette illusion, une approche anthropologique de la perspective devient possible, en trois étapes. Il s'agit d'abord de comprendre l'illusion du mouvement (et donc de la vie) en termes d'agentivité, et donc d'explorer la relation imaginaire qui s'instaure nécessairement entre une ouvre d'art et son observateur. La deuxième étape concerne la définition $\mathrm{du}$ concept d'agentivité. La grande majorité des historiens de l'art indique par ce terme une sorte de "substance indifférenciée ", quelque chose qui serait, en soi et par définition, propre au " pouvoir " de l'image. Pour l'anthropologue, il faut au contraire étudier les conditions d'existence de l'agentivité d'une image et identifier l'ensemble de relations qui l'engendrent. La compréhension du processus d'attribution d'une agentivité à l'œuvre d'art implique donc de reconnaître, dans l'image elle-même, une forme de pensée qui permet d'y projeter une subjectivité. Il faut donc étudier l'anthropomorphisme du regard, pour reprendre l'expression de Warburg, comme une forme de pensée inhérente à l'image, et non pas comme un phénomène cognitif pré-rationnel de base ${ }^{8}$. Le troisième point (qu'on ne développera pas ici) concerne l'analyse comparative. Si l'on adopte cette approche, il devient possible de concevoir une forme élargie de comparatisme, fondée sur les formes d'agentivité propres à des œuvres qui peuvent appartenir à des époques et des cultures différentes, au sein de l'Occident et au-delà.

Mais venons-en au parer vivo. On ne peut naturellement étudier cette question sans se référer aux théories que les grands penseurs de la Renaissance italienne, Giovanni Pico della Mirandola, Marsilio Ficino, Tommaso Campanella et Giordano Bruno, ont formulé à propos du concept de vie. Sur ce point, les travaux de Frank Fehrenbach sont très éclairants, et on ne peut que lui rendre ici un chaleureux hommage ${ }^{9}$. Cela dit, les théories néoplatoniciennes ou aristotéliciennes liées à la médecine, à la logique ou à la magie naturelle ne nous offrent qu'une toile de fond, une cosmologie générale qui est loin d'épuiser le champ de la perspective. Dès que l'on approche la pratique des artistes, on voit apparaître des difficultés, des solutions et des choix qui distinguent nettement l'exercice de l'art de celui de la philosophie. La définition idéale de la notion de vie, notamment, est loin d'aller de soi quand on passe des textes des théoriciens aux moyens propres 
à la peinture et à la sculpture. Dès l'époque de Filippo Brunelleschi, elle s'élabore dans un contexte qui oppose l'acte du ritrarre (littéralement " faire le portrait » des apparences) à celui d'imiter. Dans la langue des artistes italiens de l'époque, ritrarre signifie en effet " décrire ce que l'on voit le plus exactement possible ». La technique de l'imitation idéale, dont Alberti a donné la version canonique, renvoie au contraire à l'application des règles, indépendantes de l'apparence, que la tradition antique (Vitruve en particulier) a fixées pour atteindre la beauté. Ces débats autour de la vérité de l'image font surgir l'un des premiers paradoxes du rapport de la perspective à la vérité : si l'artiste suit exclusivement la voie de la description des apparences, il reste fidèle à l'expérience de son regard mais risque de trahir son sujet. Au lieu de l'image sainte du Christ, on verra apparaître, par exemple, la figure vulgaire d'un paysan ou l'image sacrilège du cadavre d'un condamné. Au contraire, en suivant la seule voie de l'imitation, l'apparence du vivant se fera moins intense et sa figure se rapprochera du modèle, parfait mais inanimé, de la statue antique. L'artiste réalisera une figure à l'apparence correcte, mais dépourvue d'individualité, où le " mouvement intérieur de l'âme " sera à peine visible. Entre ces deux principes d'application de la perspective, qui conduisent respectivement vers le rendu d'une image idéale ou vers la mise en place d'une description fidèle à l'expérience du regard, une sorte de " polarisation " (au sens que Warburg a donné à ce terme) s'établit dans la peinture italienne. Si, dans la pratique de la peinture, ces deux choix extrêmes sont parfois perçus comme s'excluant l'un l'autre ou encore poursuivis en tant que tels ${ }^{10}$, le plus souvent, lorsque l'un des deux critères prend le dessus, l'autre ne disparaît pas tout à fait et reste latent dans l'image, sous une forme implicite. Il ne s'agit pas de conceptions poétiques caractérisant l'œuvre de tel ou tel artiste (même si cela peut aussi être le cas), mais plutôt de choix techniques qui traversent le champ de la perspective dans son ensemble.

Comment peut-on atteindre le parer vivo dans le contexte de cette opposition ? Très proche de l'enseignement d'Alberti, Andrea Mantegna chercha toujours à imiter le plus possible l'art antique. Selon Giorgio Vasari, il s'opposait si fortement au ritrarre, au "portrait " des choses réelles, qu'il alla jusqu'à préférer les statues antiques aux êtres vivants comme modèles de ses figures. Pour atteindre la perfection des apparences, il n’hésitait donc pas à s'inspirer de l'inanimé. Vasari raconte que Francesco Squarcione lui-même, qui fut son maître, critiqua ses fresques de la chapelle Ovetari à Padoue, précisément parce qu'il y avait " imité les marbres antiques, de la copie desquels on ne peut apprendre parfaitement la peinture, parce que la pierre conserve toujours sa raideur et ne peut pas rendre la mollesse des chairs et des choses naturelles (qui se plient et font divers mouvements) ${ }^{11}$ ". Vasari ajoute que Mantegna, piqué au vif par les reproches de Squarcione, persistait pourtant à croire qu'il était rare de rencontrer la perfection des formes dans la réalité. Pour atteindre l'idéal de la beauté, il fallait donc passer par la synthèse sublime des apparences que les grands artistes (les eccellenti maestri) de l'Antiquité avaient déjà opérée avant lui. Mantegna en déduisait que les statues antiques étaient infiniment "plus parfaites et plus équilibrées " que les corps réels. Quand on les comparait aux corps vivants des hommes et des femmes, elles se montraient toujours " plus précises et plus accomplies" (più tocche e terminate) même « dans les muscles, les veines et les nerfs ». Les statues étaient susceptibles de mieux illustrer non seulement la beauté idéale, mais aussi l'anatomie du corps humain. Selon Vasari, Squarcione avait bien repéré un défaut réel dans la manière de peindre de son ancien élève : peintre de grande valeur, Mantegna resta pourtant lié toute sa vie à une " manière un peu tranchante, se rapprochant plus de la pierre que de la chair vive ${ }^{12}$ ".

Aujourd'hui, personne ne doute du génie de Mantegna. Son œuvre nous semble néanmoins marquée par la recherche d'une relation, parfois paradoxale, entre l'apparence idéale des formes et le sentiment de la vie. Dans nombre de ses créations, l'action, parfois même 


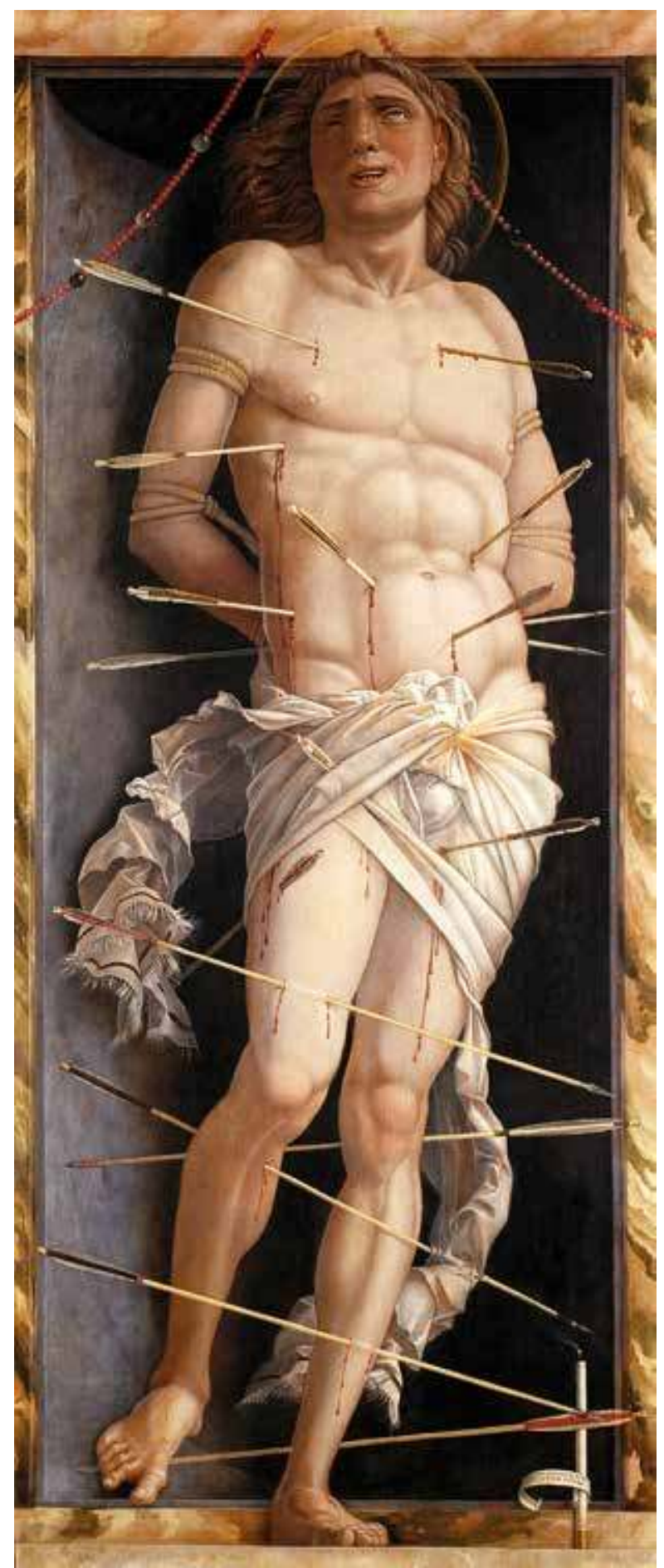

1. Andrea Mantegna, Saint Sébastien, 1490, Venise, $\mathrm{Ca}^{\prime} \mathrm{d}^{\prime} \mathrm{Oro}$, collection Franchetti. violente, est explicitement confiée à des figures en marbre ou en bronze, peintes en tant que telles. En revanche, de nombreuses figures « vivantes » semblent maintenir chez lui une telle fidélité au modèle de la statue qu'elles nous apparaissent parfois comme figées dans une sorte de fixité solennelle. Dans le Saint Sébastien de la Ca' d'Oro (fig. 1), ce jeu est poussé à son paroxysme. Percé d'un grand nombre de flèches qui semblent multiplier les points de vue sur son corps, Sébastien adresse un dernier regard vers le ciel. Ce geste ultime renvoie sans doute à l'agonie, au dernier instant vécu avant la mort. Son état transitionnel est souligné par un autre geste, plus discret : Sébastien avance son pied gauche vers l'observateur. Tout son corps est animé par ce mouvement, repris par son pied droit, qui est encore soulevé. Le point de vue est placé si bas dans la composition que la figure du saint semble s'approcher de l'observateur. La position de son épaule, tout comme celle de son coude, signale une légère torsion. Sébastien avance son pied vers un plan qui semble extérieur à l'espace de la composition. Si le saint est surpris dans l'acte de franchir un seuil, mouvement qui fait sans doute allusion au passage de la vie à la mort, la définition de l'espace est moins explicite, puisque le personnage est entouré d'un cadre de faux marbre. L'observateur qui tourne son regard vers le haut du tableau - à gauche, derrière le chapelet de corail - s'aperçoit progressivement qu'il s'agit d'une niche, lieu par excellence dans lequel, selon le modèle de l'Antiquité, on plaçait les statues. Le corps de Sébastien respecte la morphologie, savamment calculée, de la statue antique, et la blancheur de sa peau, peinte d'une couche très légère de tempera, évoque l'apparence d'un marbre gris. En même temps, l'expression de douleur extrême du visage et les traces de sang sur sa peau renvoient à un corps encore bien vivant. D'autres signes de vie, plus indirects, apparaissent. Toute la composition et l'instant qu'elle restitue sont marqués par un autre passage, à la fois symbolique et immatériel : une brise légère, qui vient de la droite - la lumière venant, quant à elle, de la gauche -, 


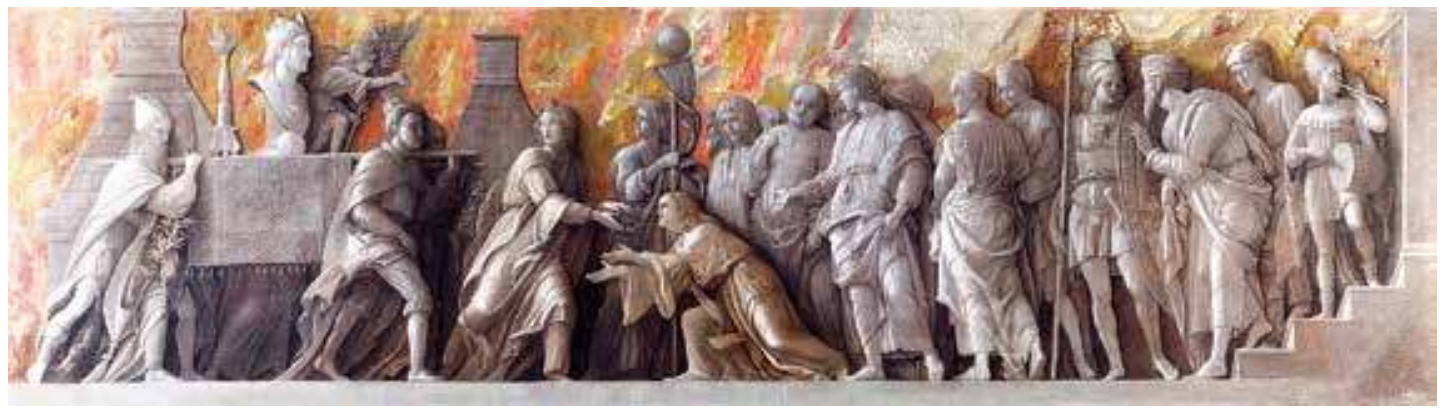

traverse le tableau. Elle soulève, de part et d'autre, le tissu blanc qui ceint les flancs du saint, et agite aussi, en haut, ses cheveux. En même temps, ces draperies sont saisies dans un état d'immobilité si parfaite, elles sont élaborées selon une plastique des volumes si classique qu'elles renvoient encore à une statue. Enfin, une dernière indication de vie apparaît. En bas à droite, Mantegna a placé une bougie sur la marche où Sébastien pose le pied gauche. Elle vient juste de s'éteindre, puisque la brise fait encore se dissiper la fumée qui en émane. Cette bougie et cette fumée nous ramènent, de nouveau, vers l'instant où le corps de Sébastien, pour la dernière fois, vibre de vie. Autour de la bougie se trouve un cartiglio où apparaissent discrètement les mots " Nihil nisi divinum stabile est, et cetera fumus " ( "Rien ne demeure, sinon le divin. Tout le reste est fumée ${ }^{13}$ "), qui confirment que cette œuvre est une méditation sur la mort imminente. À travers cette formule, que le tableau invite à lire, l'observateur se voit adresser une parole où la présence de Sébastien, statue et corps vivant, animé et inanimé, s'incarne. Il est donc possible de répondre à la question que nous avons posée : pour donner l'apparence de la vie au corps de saint Sébastien, au sein de la polarisation entre ritrarre et imitare, Mantegna l'oppose à son contraire, l'image parfaite d'une statue sans vie. Le semblant de vie qui nous frappe dans cette image n'est pas le résultat d'une relation spéculaire et, pour ainsi dire, symétrique entre une personne vivante et sa représentation. L'instant de vie qui rend le corps de Sébastien si impressionnant est le résultat d'une élaboration de traits fragmentaires et contradictoires. Une telle conclusion semble suggérer qu'une image en perspective, considérée du point de vue de la polarisation entre ritrarre et imitare, apparaît vivante si et seulement si elle apparaît à la fois comme animée et inanimée. On retrouve là un concept qui sera plus tard admirablement formulé par Michelangelo dans le poème cité en exergue à ce texte : il s'agit non seulement de donner l'image d'un corps peint comme s'il était vivant, mais aussi de la rapprocher d'une image de la mort et de la pierre inanimée. Il faut que ce soit, dit Michelangelo, " una immagine viva vicino a morte".

Cette conclusion pourrait constituer la première étape d'une série des formes possibles du parer vivo qu'on pourrait saisir, dans la longue durée du paradigme de la perspective, au sein de l'art occidental et au-delà. Dans le cadre de cet article, je souhaiterais voir si cette relation paradoxale entre l'animé et l'inanimé, identifiée dans le Saint Sébastien de Venise, est un cas isolé dans l'œuvre de Mantegna.

L'Introduction du culte de Cybèle à Rome (fig. 2) est l'une des dernières œuvres du maître, commandée en mars 1505 par la famille vénitienne des Cornaro, qui prétendait descendre de l'illustre gens Cornelia de la Rome antique ${ }^{14}$. Cette peinture montre comment le buste de Cybèle, divinité originaire d'Anatolie (à l'extrême gauche de la composition) entre 


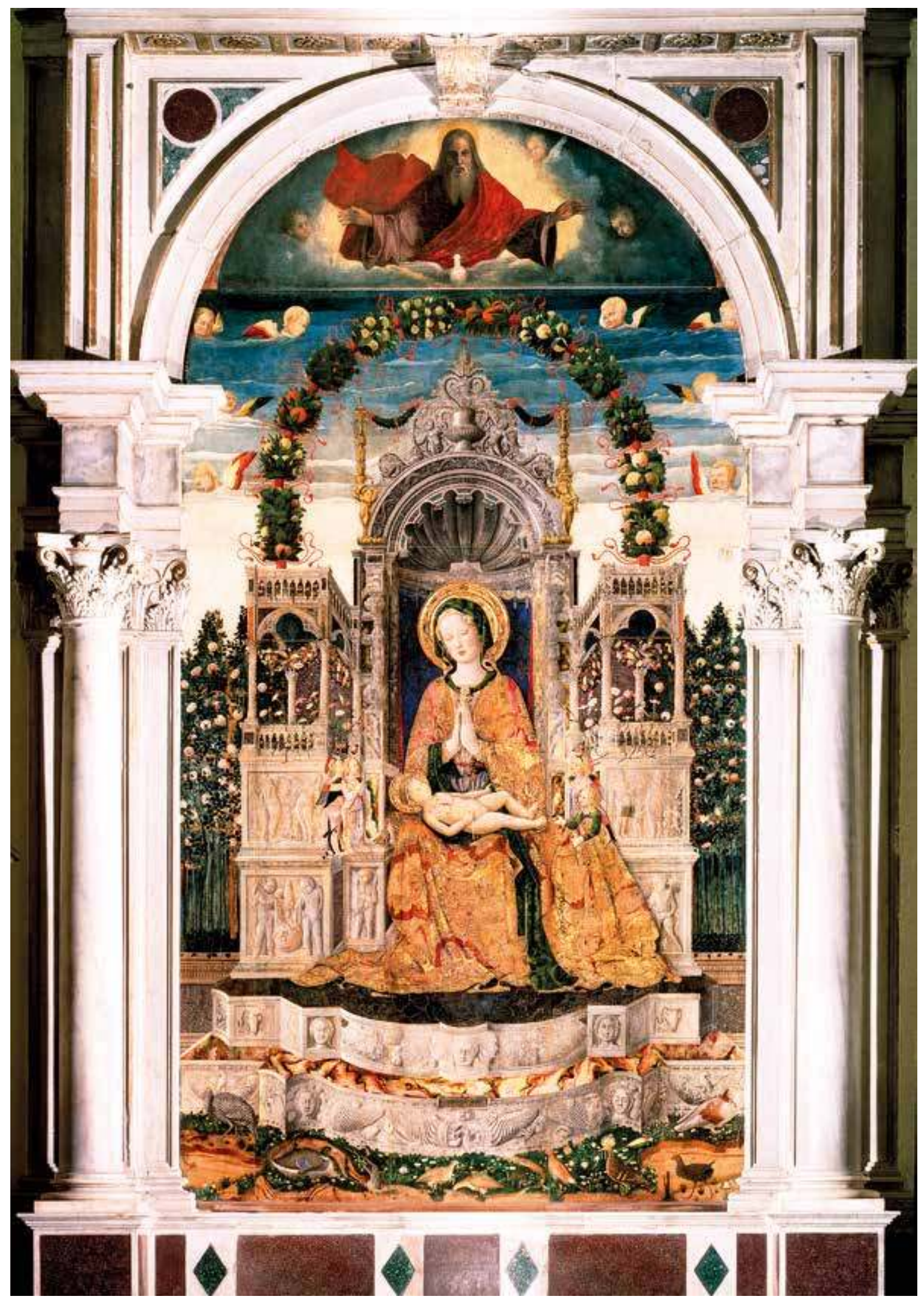


officiellement à Rome, en passant devant les tombes en forme de pyramide des ancêtres de Scipion Nasica, censé être un illustre ancêtre des Cornaro. Pour certains, Scipion est représenté par le personnage qui se tient debout à côté de la litière transportant le buste de la déesse, et semble marcher vers la droite. Pour d'autres, le Consul serait représenté par le personnage qui se trouve plus à gauche, apparemment engagé dans une conversation animée avec un autre personnage debout. $\mathrm{Au}$ centre du tableau, un personnage féminin, que l'on peut identifier avec la patricienne romaine Claudia Quinta ${ }^{15}$, semble rendre hommage à la divinité en posant un genou à terre devant elle ${ }^{16}$. Si l'on en croit les sources littéraires qui semblent être à l'origine de ce sujet singulier et très rarement représenté, Claudia avait joué un rôle crucial pour faire venir la déesse à Rome. Selon Ovide, elle avait sauvé d'un naufrage le bateau sur lequel sa statue voyageait lors de son entrée dans le port d'Ostie. Claudia fait partie, avec le personnage debout à sa gauche et la figure qui représente peut-être Scipion à sa droite, du groupe central de la composition : ces personnages mettent en scène l'action

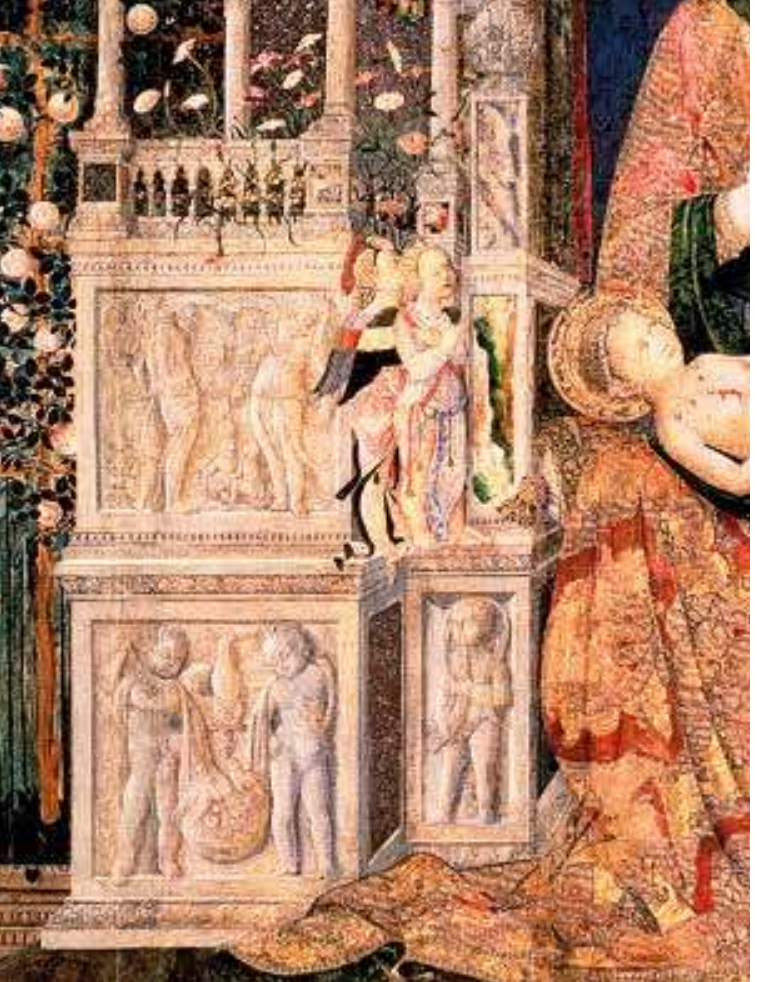

3a-b. Antonio da Negroponte, La Vierge sur le trône adorant l'Enfant Jésus, et un détail [b], 1470, Venise, San Francesco della Vigna. cérémonielle et s'adressent directement à la divinité. À droite, les autres figures indiquent cette action d'un geste, observent ou commentent entre elles l'arrivée triomphale de Cybèle, ou encore se préparent à jouer en son honneur, sans entrer en relation avec elle.

Tous les auteurs qui ont travaillé sur ce tableau ont reconnu que la singularité de L'Introduction du culte de Cybèle n'est pas seulement liée à la source, antique et très rare, qu'elle met en scène. Ce tableau témoigne aussi d'un degré de mimêsis picturale du bas-relief romain rarement atteint en peinture. Le sujet de la peinture de Mantegna n'est donc pas seulement son modèle littéraire. C'est aussi la représentation, techniquement parfaite, d'un bas-relief romain, comme le signale, tout le long de la composition, l'arrière-plan qui simule un marbre rouge sur lequel campent les figures grises, parce que supposées sculptées dans la pierre, des personnages. La question de la relation entre peinture et sculpture est un des grands thèmes de l'art italien du Quattrocento, et il est naturellement impossible de la reprendre ici dans toute sa complexité. Je retiendrai seulement deux exemples de cette relation. Autour de 1435, Donatello avait conçu des images hybrides, combinant sculpture et peinture. À San Lorenzo, à Florence, il avait juxtaposé des figures modelées en stuc blanc à des fonds peints où apparaissaient des architectures ou des paysages. Comme Podro l'a remarqué ${ }^{17}$, Donatello donnait ainsi à voir des compositions où des images qui répondaient à la lumière naturelle alternaient avec des scènes peintes, où seule l'imagination pouvait projeter une lumière. C'était là un cas exceptionnel et très discuté. À l'époque, il existait des manières plus simples de concevoir cette relation : insérer, ainsi, à l'intérieur d'un tableau, des monochromes 
présentés comme faisant partie d'un bas-relief antique est une stratégie picturale assez répandue dans l'Italie du Quattrocento. On trouve dans la Vierge et l'enfant d'Antonio da Negroponte (fig. 3a-b), aujourd'hui dans l'église San Francesco della Vigna, à Venise, un exemple de cette manière d'établir une comparaison visuelle, tout à fait proche de l'œuvre et de l'enseignement de Mantegna.

Dans ce tableau, Negroponte établit un rapport d'analogie et de contraste entre les anges vivants qui se trouvent des deux côtés de l'enfant Jésus et leurs équivalents sculptés, comme dans un bas-relief antique, sur le trône monumental où la Vierge est assise. Du point de vue des humanistes et des théoriciens de la peinture, c'était là une manière de comparer un thème d'inspiration chrétienne avec un thème antique, en représentant ainsi une relation de filiation entre le présent dans lequel la scène se situe et le passé de

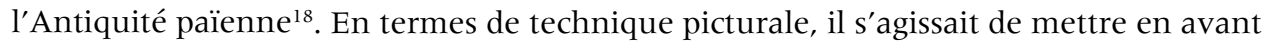
un contraste entre la lumière et la couleur qui caractérisent les êtres doués d'une vie, d'une part, et, d'autre part, la grisaille sans couleur et sans contraste du bas-relief, par définition dépourvue de lumière. Dans L'Introduction du culte de Cybèle à Rome, Mantegna opère un changement radical. Il peint une istoria où tous les personnages sont présentés comme faisant partie d'un bas-relief antique. Si l'on suit la scène de gauche à droite, toutes les figures sont monochromes. Le jeu de la lumière naturelle sur les plis de la pierre sculptée est représenté d'une manière extrêmement soignée, par des touches fines de peinture blanche. Scipio, les sénateurs, les musiciens qui s'apprêtent à jouer pour célébrer la déesse sont donc des statues qui, en reflétant la lumière, offrent des contrastes composés de blanc et de gris. Seule exception à cette règle, la figure agenouillée de Claudia Quinta qui, recevant la lumière, est immergée dans la couleur chaude, entre rouge et ocre, d'un corps vivant (fig. 4). Par ce véritable coup de théâtre visuel, à la fois très subtilement et très clairement indiqué, Mantegna ne joue pas seulement sur une comparaison entre peinture et sculpture, ou entre la couleur de l'être vivant et le gris de la pierre. Le peintre reprend, dans ce tableau, le défi lancé avec le Sébastien de la Ca' d'Oro : identifier des termes intermédiaires entre statue et corps vivant. Il s'agit d'abord d'atteindre, dans le modelage des figures du bas-relief, une imitatio géométriquement parfaite de l'idéal classique de la beauté. À partir de là, deux indications contradictoires sont ajoutées. D'une part, Mantegna attribue à ces figures pourtant inanimées des actions très éloquentes et, d'autre part, il les immerge dans une lumière naturelle qui provient de l'extérieur. Il applique donc une description très picturale, et qui mime la présence de la lumière naturelle, non pas à des figures vivantes, mais à des images sculptées. Dans L'Introduction du culte de Cybèle, ces effets de lumière sont poursuivis dans le moindre détail, non seulement dans les drapés des toges dont les personnages sont habillés, mais jusque dans la description microscopique du tapis qui pend du baldaquin portant Cybèle - qui se révèle donc léger et minutieusement tissé, bien que composé en pierre. La lumière rend donc "presque vivantes " les figures en pierre qui composent le bas-relief.

Ce jeu, qui se fonde déjà sur le paradoxe d'une matière à la fois inanimée et vivante, s'intensifie brusquement autour de la figure de Claudia Quinta. Comparons Claudia avec le Sébastien de la Ca' d'Oro. À Venise, pour représenter le corps de Sébastien, Mantegna choisit de partir de l'idéal de la statue dans une niche et cherche des termes intermédiaires qui puissent la mettre en rapport avec un corps vivant. Dans son bas-relief peint, Mantegna inverse le jeu. Claudia Quinta, qui devrait être une figure sculptée comme les personnages qui l'entourent, prend subitement les tons chauds de la lumière naturelle et apparaît ainsi, parmi les statues grises, comme une femme vivante. Claudia est une statue cueillie dans l'instant où elle se révèle vivante. Dans ce sens, elle apparaît comme la figure inversée du Sébastien de Venise, qui met en scène un corps vivant et souffrant, saisi dans l'instant 
où il cède à la mort et prend l'apparence grise et immobile d'une statue dans sa niche. Ce jeu entre vie et mort, confié à la lumière, entraîne aussi une autre conséquence, qui concerne l'espace et le temps. Le jeu complexe que Mantegna joue entre statue et corps vivant affecte la relation entre espace et temps exprimée ici par la peinture. La figure de Claudia ne se révèle pas vivante seulement parce qu'elle réagit par la couleur à la lumière qui la frappe, à la différence des autres personnages, qui restent gris et figés. Elle est vivante aussi parce qu'elle est, pourrait-on dire, représentée au présent. Le rayon de lumière qui l'investit marque un instant dans le présent de la scène. Nous avons vu que Mantegna a recours à la peinture pour obtenir une apparence de vie, à travers l'apparition d'une couleur chaude que la sculpture ne peut atteindre. Nous voyons maintenant que ce sentiment de vie naît d'un double constat, qui concerne aussi bien l'espace que le temps de l'image. Claudia est vivante parce qu'elle n'appartient plus à un passé qu'il s'agit de célébrer ou de conserver en mémoire. Elle est là, devant l'observateur, à l'instant même où le rayon de lumière l'atteint. C'est cette lumière qui la rend unique dans un espace (et un temps) où les autres figures, face au même rayon, restent prisonnières des nuances raffinées d'un gris, de l'inanimé tout autant que du passé.

Nous découvrons donc que le parer vivo - l'apparence de la vie propre à la représentation en perspective - ne doit rien à la géométrie. Il s'explique plutôt comme la mise en place, dans ce cadre, de l'intuition d'un lien, opéré par le regard, entre le sujet d'une représentation, son destinataire et, indirectement, son auteur. Dans son remarquable Traité de l'évidence, Fernando Gil a proposé une définition du concept d'évidence qui peut nous être utile pour mieux comprendre ce point. Selon Gil, il faut distinguer deux aspects de ce concept : le premier, s'appuyant sur l'équivalence entre «voir "

4. Andrea Mantegna, Claudia Quinta s'agenouille devant Cybèle, détail de L'Introduction du culte de Cybèle à Rome, Londres, The National Gallery.

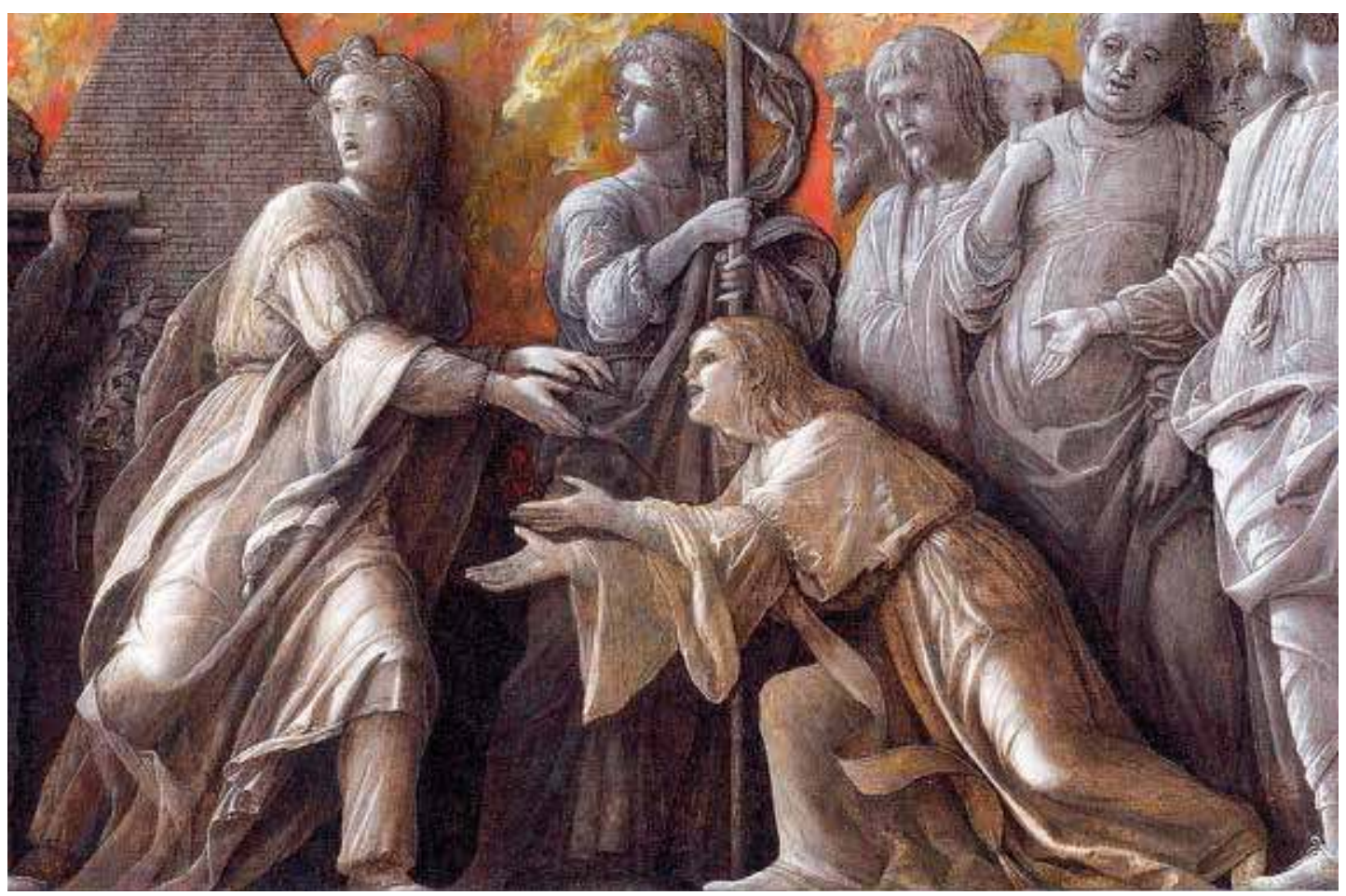


et " connaître " qui est devenue traditionnelle au sein de notre culture, s'applique au discours (mathématique, par exemple) et désigne la connaissance "intuitive " et immédiate d'un phénomène. Le second repose, au contraire, sur l'idée de regard actif, compris comme un moyen d'établir un contact visuel, une relation à autrui. Or, l'intuition du lien que le "semblant de vie " réalise au sein de la perspective opère une synthèse entre ces deux aspects de l'évidence. À travers l'illusion spatiale, la perspective réunit une certaine épistémologie du regard - la vérité de ce que l'on voit, en appréhension immédiate - avec la notion, liée aux idées d'exercice actif du regard et de contact visuel, d'établissement d'un lien entre l'image et celui ou celle qui la regarde. Dans les deux œuvres étudiées dans ces pages, le travail de Mantegna consiste non seulement à matérialiser une bonne illusion, au sens technique du terme, mais aussi à transformer l'illusion de l'espace en évidence du lien. Là réside la racine de la vie de l'image, et du mouvement implicite qui l'anime. 


\section{Carlo Severi}

Directeur d'études à l'École des hautes études en sciences sociales et directeur de recherche au CNRS, Carlo Severi est membre du Laboratoire d'anthropologie sociale du Collège de France depuis 1985. Il a été Getty scholar au Getty Institute for the History of Art and the Humanities de Los Angeles (1994-1995 et 2017), fellow du Wissenschaftskolleg de Berlin (2002-2003), visiting fellow au King's College de l'Université de Cambridge (2012-2013) et Richard Lehmann visiting professor à la Villa I Tatti (Center for Renaissance Studies, Harvard University, 2018 et 2019) à Florence.

\section{NOTES}

1. Michelangelo Buonarroti, Rime, publié dans Rime e Lettere, Paola Mastrocola (éd.), Turin, UTET, 1992, N. 241, p. 207. Selon Frey, ce sonnet a été composé en 1542 .

2. «Poi moverà l'istoria l'animo quando gli uomini ivi dipinti molti porgeranno suo proprio movimento d'animo [...] ma questi movimenti d'animo si conoscono dai movimenti del corpo. " Leon Battista Alberti, De Pictura (1435), Cecil Gayson (éd.), https://books. apple.com/fr/book/de-pictura/id768016010, 1998, p. 48, paragraphe 41 .

3. "Dicesi vivere il corpo quando a sua posta abbia certo movimento ", Alberti, 1998, cité n. 2, p. 44, paragraphe 37.

4. Carlo Severi, "Anthropologie de l'art ", dans Pierre Bonte et Michel Izard, Dictionnaire de l'ethnologie et de l'anthropologie, Paris, Presses universitaires de France, 1991, p. 81-85.

5. Carlo Severi, L'objet-personne : anthropologie de la croyance visuelle, Paris, Éditions Rue d'Ulm / Musée du quai Branly, 2017.

6. Aby Warburg, Fragments sur l'expression, Paris, Éditions de l'Écarquillé, 2015.

7. Voir Ernst Gombrich, Art and Illusion. A Study in the Psychology of Pictorial Representation, Londres, Phaidon, 1977 et Thomas Kuhn, La Tension essentielle. Traditions et changements dans les sciences, Paris, Gallimard, 1990 [éd. orig. : The Essential Tension. Selected Studies in Scientific Tradition and Change, Chicago, Chicago University Press, 1977].

8. Sur ce point, voir David Freedberg et Vittorio Gallese, "Motion, Emotion and Empathy in Esthetic Experience", dans Trends in Cognitive Science, vol. 11, $\mathrm{n}^{\circ}$ 5, 2007, p. $197-203$.

9. Voir par exemple Frank Fehrenbach, "Quasi animata forma. Living Art in the Early Modern Art ", dans Marc Wellmann, BIOS: Konzepte des Lebens in der zeitgenössischen Skulptur / BIOS: concepts of life in contemporary sculpture, cat. exp. (Berlin, Georg-Kolbe-Museum, 2012), Cologne, Wienand, 2012, p. 30-39. "Coming Alive: Some Remarks on the rise of "Monochrome"
Sculpture in The Renaissance », dans Source: Notes in the History of Art. Special issue "Superficial? Approaches to Painted Sculpture ", vol. 30, 2011, n 3, p. 47-55, et son entrée "Lebendigkeit », dans le dictionnaire dirigé par Ulrich Pfisterer (dir.), Metzler-Lexikon Kunstwissenschaft, Stuttgart, 2003, p. 222-227. Un certain nombre d'essais de cet auteur sont aujourd'hui réunis dans Frank Fehrenbach, Quasi vivo. Lebendigkeit in der italienischen Kunst der Frühen Neuzeit, Berlin / Boston, De Gruyter, 2020.

10. Sur ce point, voir Carlo Severi, 2017, cité n. 5, p. 298302.

11. Giorgio Vasari, Vies des artistes : vies des plus excellents peintres, sculpteurs et architectes, Léopold Leclanché (éd.), Paris, Grasset, 2007, p. 162 [éd. orig. : 1550].

12. Ibid., p. 163.

13. Traduction proposée par Jacques Darriulat lors d'une conférence prononcée à l'auditorium du Louvre, le 27 octobre 2008, dans le cadre de l'exposition «Mantegna » (2008-2009).

14. Giovanni Agosti, "Verso la maniera moderna », dans Giovanni Agosti et Dominique Thiébault, Mantegna, cat. exp. (Paris, musée du Louvre, 2008-2009), Rome, Officina Libraria, 2008, p. 410-411. Mauro Lucco, Mantegna, Milan, 24 Ore, 2013, p. 360-361.

15. Neville Rowley évoque une contamination de passages empruntés à Live, Ovide et Appien (Neville Rowley, "The Last Works », dans Caroline Campbell et al. (dir.), Mantegna and Bellini. A Renaissance Family, Londres, National Gallery Company, 2018, p. 250. Agosti (ibid.) renvoie aussi à la Roma Triumphans de I'humaniste Flavio Biondo. Sur l'histoire de Claudia Quinta, voir aussi Sabine Blumenröder, Andrea Mantegna, Die Grisaillen. Malerei, Geschichte und Antike Kunst im Paragone des Quattrocento, Berlin, Gebr. Mann Verlag, 2008, p. 26-37.

16. La fiche anonyme du catalogue en ligne de la National Gallery de Londres confirme cette identification : «Mantegna entremêle plusieurs sources littéraires pour créer cette image. La femme que l'on voit s'agenouiller face à la litière pourrait être Claudia Quinta [Mantegna wove together a number of literary sources to create the image. The woman shown wailing on her knees in front of the litter may be Claudia Quinta]. » Signalons toutefois que Rowley (ibid., p. 255) écrit à ce propos que « la procession est accueillie par des dignitaires romains, dont Scipion lui-même, bien qu'il n'y ait pas de consensus quant à son identification dans la figure agenouillée en signe de dévotion devant elle, ou I'homme debout plus loin à droite, esquissant un geste du bras droit [the procession is greeted by Roman dignitaries, one of them must be Scipio himself, though there is no consensus as to whether he is the figure kneeling in fervent welcome before it, or the man standing further to the right, gesturing with his right arm] ». Agosti, pour sa part (ibid., p. 410) semble croire que Scipio est la figure agenouillée, sans proposer aucune identification de la figure debout à côté d'elle ni de celle qui, selon la fiche du catalogue de la National Gallery, représenterait, à droite, Scipion Nasica en tant qu'hôte officiel de la déesse anatolienne. Rappelons aussi que les sources parlent de Scipio comme d'un illustre sénateur, dont le prestige et l'autorité sont si 
établis que le Sénat l'autorise à héberger chez lui une divinité. Difficile donc de le reconnaître dans la figure agenouillée ; il s'agirait plutôt d'une femme ou d'un jeune homme.

17. Michael Podro, Depiction, New Haven, Yale University Press, 1998, p. 49-51.

18. Salvatore Settis et Carlo Ginzburg ont interprété dans ce sens l'insertion d'un bas-relief antique représentant le transport du corps de Méléagre défunt (mentionné par Alberti dans le livre II du De Pictura, cité n. 2, p. 48) dans le Compianto di Cristo de Luca Signorelli à Orvieto. Voir Maria Luisa Catoni (dir.), Tre Figure Achille, Meleagro, Cristo, Milan, Feltrinelli, 2013, p. 88 et 121-122. 